\title{
Notices and Announcements
}

\section{$\int_{Q A B}$}

\section{Society for the Quantitative Analyses of Behavior}

SAB holds its annual meeting in conjunction with the Association for Behavior Analysis this year at the Walt Disney World Dolphin Hotel in Orlando, Florida on Friday and Saturday, May 22-23, 1998. Talks focus on the development and use of mathematical formulations to characterize behavior.

This year presentations will be given by Robert Allan; Russell Church; Michael Davison \& William Baum; Valentin Dragoi; William K. Estes; Armando Machado; James MacDonall; William Hutchison; Ralph Miller \& Hernan Savastano; Peter Killeen \& Lewis Bizo; John Staddon; Richard S. Sutton; and John Wearden. fQAB is also sponsoring Preeminent Tutorials by William Baum, Steven Hursh, John Nevin, and John Wixted.

Registration' fees will be waived for students presenting quantitative papers or posters at ABA or $\int_{\text {QAB. }}$ Visit our Web page for abstracts and additional information. For information, registration or poster submissions, contact:

William L. Palya, אQAB Program Chair

Jacksonville State University, Jacksonville, AL 36265

http://www.jsu.edu/psychology/sqab.html

palya@sebac.jsu.edu 205-782-5641 (voice) 205-782-5680 (fax) 